\title{
$\beta$-diversity scaling patterns are consistent across metrics and taxa
}

Laura H. Antão ${ }^{1,2}$, Brian McGill ${ }^{3}$, Anne E. Magurran ${ }^{1}$, Amadeu Soares ${ }^{2}$, Maria Dornelas ${ }^{1}$

${ }^{1}$ Centre for Biological Diversity, University of St Andrews, Sir Harold Mitchell Building, St Andrews,

Scotland, UK

${ }^{2}$ Department of Biology and CESAM, Universidade de Aveiro, Campus Universitário de Santiago,

Portugal

${ }^{3}$ School of Biology and Ecology, Sustainability Solutions Initiative, University of Maine, Orono, ME 04469, USA

Corresponding author: Laura H. Antão, Centre for Biological Diversity, Sir Harold Mitchell Building, University of St Andrews. KY16 9TF, St Andrews, Scotland, UK. E-mail: liha@ st-andrews.ac.uk

Decision date: $05-\mathrm{Dec}-2018$

This article has been accepted for publication and undergone full peer review but has not been through the copyediting, typesetting, pagination and proofreading process, which may lead to differences between this version and the Version of Record. Please cite this article as doi: [10.1111/ecog.04117]. 


\section{Abstract}

$\beta$-diversity (variation in community composition) is a fundamental component of biodiversity, with implications for macroecology, community ecology and conservation. However, its scaling properties are poorly understood. Here, we systematically assessed the spatial scaling of $\beta$-diversity using 12 empirical large-scale datasets including different taxonomic groups, by examining two conceptual types of $\beta$ diversity and explicitly considering the turnover and nestedness components. We found highly consistent patterns across datasets. Multiple-site $\beta$-diversity (i.e. variation across multiple sites) scaling curves were remarkably consistent, with $\beta$-diversity decreasing with sampled area according to a power law. For pairwise dissimilarities, the rates of increase of dissimilarity with geographic distance remained largely constant across scales, while grain size (or scale level) had a stronger effect on overall dissimilarity. In both analyses, turnover was the main contributor to $\beta$-diversity, following total $\beta$-diversity patterns closely, while the nestedness component was largely insensitive to scale changes. Our results highlight the importance of integrating both inter- and intraspecific aggregation patterns across spatial scales, which underpin substantial differences in community structure from local to regional scales.

Keywords: species composition; dissimilarity; diversity partitioning; nestedness component; spatial turnover; scale dependence; intraspecific aggregation; spatial grain 


\section{Introduction}

Biodiversity patterns and the mechanisms driving them are inherently scale dependent (Wiens 1989 , Levin 1992, McGill 2010a, McGill et al. 2015). Multiple processes determine the distribution and abundance of species, acting upon ecological communities differently at different scales, e.g. climate, local environmental conditions, dispersal and species interactions (MacArthur 1972, Ricklefs 1987, McGill 2010a). Beta ( $\beta$ ) diversity quantifies the variation of species composition between assemblages or sites (Whittaker 1960). It is a fundamental component of biodiversity, with implications for community ecology, macroecology and conservation (Whittaker 1960, Anderson et al. 2011, Socolar et al. 2016). As additional habitat types and different environmental features are included in larger geographical areas, $\beta$ diversity patterns are expected to be scale dependent (Koleff et al. 2003, Tuomisto 2010a, Barton et al. 2013). However, while the scaling properties of species richness have received substantial attention (e.g. Rosenzweig 1995; Harte et al. 2009; Storch et al. 2012), the scaling of $\beta$-diversity is less understood, with a lack of theoretical predictions about the form of $\beta$-diversity scaling patterns (Koleff et al. 2003, Gaston et al. 2007, Barton et al. 2013).

Analyses of $\beta$-diversity can be used to address two sets of questions: variation among sites within a given spatial extent, habitat type or experimental treatment (multiple-site $\beta$-diversity) vs differences between sites along a spatial or environmental gradient (pairwise dissimilarities), thus yielding two conceptual types of $\beta$-diversity (Anderson et al. 2011). The first approach derives aggregate measures of $\beta$-diversity across all sites regardless of their location (i.e. not spatially explicit), while the second approach typically regresses pairwise site comparisons $v s$ geographic distance (distance-decay relationship (DDR); Nekola \& White 1999; Morlon et al. 2008). DDR are often well fitted by exponential or power law curves, and $\beta$ diversity is then summarized as the rate of change from this curve (Nekola and McGill 2014). Additionally, $\beta$-diversity may reflect two underlying phenomena - turnover and nestedness. The turnover component represents the replacement of species between sites, whereas the nestedness-resultant component occurs due to changes in species richness - sites with fewer species are strict subsets of richer sites (Harrison et al. 1992, Koleff et al. 2003, Baselga 2010). Nestedness and turnover are generated by different processes - species loss and species replacement respectively - therefore quantifying their relative contribution can provide insights into the mechanisms underlying $\beta$-diversity across spatial scales

'This article is protected by copyright. All rights reserved.' 
(Baselga 2010, Svenning et al. 2011). However, little is known about how the two components change across different scales. Here, we examine both multiple-site and pairwise $\beta$-diversity, as well as their components, taking advantage of the framework developed by Baselga for presence-only data (2010), in order to understand the scaling properties of $\beta$-diversity.

Expectations for the scaling of $\beta$-diversity

The measurement of $\beta$-diversity is affected by the spatial scale of observation, both in terms of grain and extent (Preston 1948, Wiens 1989, Nekola and White 1999, Mac Nally et al. 2004, Qian 2009, Keil et al. 2012, Steinbauer et al. 2012, Barton et al. 2013, Nekola and McGill 2014). For small grain sizes compared to the overall study extent, even close sampling units might be very dissimilar in their species composition, due to stochastic sampling effects and high variability in species occupancy patterns. As grain size increases, mean environmental variability decreases as a result of spatial averaging, and the probability of detecting more rare species increases (Wiens 1989, Levin 1992, Gaston et al. 2007, Keil et al. 2012, Barton et al. 2013). Hence, multiple-site $\beta$-diversity is expected to decrease as grain increases.

But what is the functional form of this relationship? And are the patterns system or taxon specific (Barton et al. 2013)? Our analysis provides the first attempt to build empirical $\beta$-diversity scaling curves - akin to the triphasic Species-Area Relationship (SAR; Williams 1943; Rosenzweig 1995; Storch et al. 2012), and the first assessment of the generality of these scaling patterns.

Pairwise dissimilarities are also expected to decrease as grain size increases, but previous studies have reported conflicting results. For instance, lower DDR rates (Nekola and White 1999) and lower $\beta$ diversity (Keil et al. 2012) were reported for larger grains. In contrast, Morlon et al. (2008) found that grain size only affected rates of decay at the smallest grain analysed, while no consistent trend was found by both Steinbauer et al. (2012) and Zacaï et al. (2016). This suggests that the influence of grain size on DDR cannot be easily predicted, and might be context and/or taxa dependent. However, these studies have all employed different approaches, varying total extent and/or grain, and analysing the patterns at different spatial scales (continents to plots). Hence, employing a systematic multiscale approach across multiple taxa can help disentangle scale effects and ecological patterns. 
A few studies have analysed turnover and nestedness components' patterns at large spatial extents for specific taxa, with their relative contributions being apparently contingent on the context and scale investigated (Baselga 2010, Svenning et al. 2011, Wen et al. 2016), but a general investigation of turnover and nestedness relative contributions independent of latitudinal, longitudinal or environmental gradients is still lacking. Turnover can be expected to be lower between larger sampled areas and for more vagile organisms. It may be that the nestedness component is less relevant between smaller sampled areas, where turnover may be the dominant driver of $\beta$-diversity. On the other hand, nestedness could also represent a smaller portion of $\beta$-diversity in scenarios with high dispersal rates. Thus, it is not clear how grain could affect the relative importance of the two components, with possible interactions resulting from other mechanisms, such as metacommunity dynamics (Leibold et al. 2004, Si et al. 2015, Tonkin et al. 2016, Schuler et al. 2017, Gianuca et al. 2017).

Here, we establish a scale gradient within 12 communities encompassing different taxa to systematically assess $\beta$-diversity scaling patterns. We undertake the first empirical assessment of multiple-site $\beta$ diversity scaling with sampled area. Additionally, we consistently investigate the behaviour of pairwise dissimilarities across the scale gradient, by testing the hypothesis that DDR rates become steeper (more pronounced) as grain size decreases. Finally, we test whether turnover decreases with area sampled, while exploring the behaviour of the nestedness component. 


\section{Material and Methods}

To quantify community dissimilarity, we used the additive partition framework proposed by Baselga (2010), where the Sørensen index represents total $\beta$-diversity accounting for all aspects of compositional variation, the Simpson index represents turnover, i.e. species replacement independent of species richness gradients, and their difference represents a measurement of dissimilarity due solely to species richness differences, i.e. the nestedness-resultant component:

$\beta_{\text {Sørensen }}=\beta_{\text {Simpson }}+\beta_{\text {Nestedness-resultant }}$.

We follow the same notation as Baselga (2010), using upper-case letters for the multiple-site metrics $\left(\beta_{\mathrm{SOR}}, \beta_{\mathrm{SIM}}, \beta_{\mathrm{NES}}\right)$, and lower-case letters for pairwise dissimilarities $\left(\beta_{\mathrm{sor}}, \beta_{\mathrm{sim}}, \beta_{\mathrm{nes}}\right)$. We emphasize that we examine the component of $\beta$-diversity due to differences in species richness, not nestedness itself (Baselga 2010, 2012). Gamma diversity (i.e. the total number of species sampled in the overall extent) was assumed constant for each dataset, and because our goal was not to estimate latitudinal gradients of $\beta$-diversity, we did not employ a null model approach (Tuomisto 2010b, Ulrich et al. 2016, Socolar et al. 2016). All analyses used presence-only data and were performed using the statistical software R (R Core Team 2017).

\section{Empirical Data}

We analysed 12 datasets comprising different taxa, namely birds, fish, benthos and trees. We selected 11 datasets from the BioTIME database (Dornelas et al. 2018) with spatial extent larger than $150000 \mathrm{~km}^{2}$ and for which the unique sampling locations were distributed across the study area so that the random splitting of the total extent would not result in portions without sampling locations (see next section). We used data from a single year for each dataset (the year with the most and more evenly distributed sampling locations). We also analysed the Forest Inventory and Analysis Database (FIA; http://fia.fs.fed.us/; USDA Forest Service 2010; Woudenberg et al. 2010), as we wanted to include tree community data in our analysis; we obtained these data using the EcoData Retriever (http://dataretriever.org; Morris and White 2013, McGlinn and White 2015). These empirical datasets cover a wide 
range of sampling grains ( 0.0001 to $400 \mathrm{~km}^{2}$ ) and total spatial extents (167 455 to $16663141 \mathrm{~km}^{2}$ ). The full list of datasets and sources can be found in Table 1.

Implementing the scale gradient

We established a scale gradient by using the fixed extent of the study area and systematically partitioning this area into smaller portions, thus varying "grain" size as follows. We drew a circle encompassing all the sampling locations from the community data and centred on the centroid of the sampling locations. A random point from the circle was selected to split the circle into halves, thirds, quarters, eights and sixteenths, using the initial random point from the bisection as reference (Fig. 1). We subsampled to obtain an equal number of samples in each subsection and pooled species abundances across the retained samples within each section. We then calculated $\beta$-diversity metrics at each level. Each study was randomly split ten times, to assess the generality of our results focusing on effects of scale independently of latitudinal or longitudinal effects. Finally, we calculated $\beta$-diversity between all the individual samples, representing the lowest level of the scale gradient. This procedure thus yielded six scaling levels covering several orders of magnitude (we were not able to establish more levels after splitting the extent into 16 sections since the sampling locations were not evenly distributed across the extents, which would lead to having empty sections or sections containing only one or two samples).

The sample areas created as described above were not exhaustively sampled (which would be a logistical impossibility). Thus, we calculated the area of the sample in two ways, bracketing the upper and lower bounds of what could reasonably be called the sampled area. First, the areas sampled were calculated using convex hull polygons encompassing the sampling locations within each section, using package rgeos (Bivand and Rundel 2016) (Fig. 1). At each level, sampled area was estimated as the minimum of the convex hull areas - the estimated metrics are representative of the smallest area. Additionally, we calculated area sampled as grain size * number of samples, representing the actual area sampled. For the lowest level of the scale gradient, we considered the grain size of individual samples, assuming a value from a similar study if the exact information was not available (Table 1). Since the range of grains analysed covers several orders of magnitude, our results are not contingent on the exact grain size at the smallest scale. Geographic distances were calculated in $\mathrm{km}$ as the distance between the centroids of each 
section, and between all the sampling locations for the grain level, using package sp (Pebesma and Bivand 2005) (Fig. 1).

\section{Multiple-site $\beta$-diversity}

To assess how multiple-site dissimilarity varied with grain, we used function beta.sample() in betapart package (Baselga and Orme 2012, Baselga et al. 2017) to calculate total $\beta$-diversity, turnover and nestedness components. beta.sample() randomly selects a specified number of sites to generate distributions of the multiple-site dissimilarity measures, thus allowing to compare the different scaling levels using an equal number of sites. To fully explore scaling, we sampled two sites at each level, drawing 1000 random samples. We fitted two models to the multiple-site $\beta$-diversity scaling curves. First, we used generalized nonlinear least squares to fit a power law to each metric as a function of area (i.e. Dissimilarity 1-( $\left.\mathrm{a}^{*} \operatorname{Area}^{\wedge}{ }^{\mathrm{b}}\right)$ ), using function gnls() from the nlme package (Pinheiro et al. 2016). Second, we fitted a linear model to the logit transformation of dissimilarity values as a function of $\log 10$ (Area). To illustrate that the patterns are not contingent on using estimates from a single trial, we compared the median $\beta_{\text {SOR }}$ values across all the trials (excluding the last one) with the values used in the analysis.

\section{Pairwise $\beta$-diversity}

We used function beta.pair() in betapart (Baselga and Orme 2012, Baselga et al. 2017) to obtain pairwise dissimilarities between the sections at each scaling level and between all the sampling locations for the grain level (pairwise dissimilarities represent the opposite of distance-decay of similarity). For the datasets with over 5000 individual samples, we randomly sampled 2000 samples for the grain level models, due to the very high number of pairwise comparisons (Table 1). We fitted negative exponential models to each dissimilarity metric $v s$ geographic distance (Nekola and McGill 2014) via GLM using the decay.model() function from betapart, which also implements a permutation test to assess significance ( $\mathrm{n}=100)$ (Baselga et al. 2017, Gómez-Rodríguez and Baselga 2018). We tested if the slopes and intercepts increased as grain decreased by bootstrapping the parameters using the function boot.coefs.decay() in betapart (1000 bootstraps; for the grain level only 100 bootstraps were used due to the very high number of calculations to perform). Finally, we assessed if coefficients for turnover $\left(\beta_{\text {sim }}\right)$ were higher than for 
nestedness $\left(\beta_{\text {nes }}\right)$ within each scaling level by calculating the probability of obtaining the opposite result by chance, by comparing the bootstrap parameter distributions. These procedures were not performed for the bisection, $1 / 3$ and $1 / 4$ levels due to the small number of pairwise comparisons available to accurately assess DDR.

\section{Results}

\section{Multiple-site $\beta$-diversity}

Multiple-site $\beta$-diversity scaling curves were very similar across the communities analysed, with $\beta_{\text {SOR }}$ decreasing with sampled area according to a power law (Fig. 2; Table S1). As these patterns were consistent across the ten random splitting trials (Supplementary Figs. S1 and S2), we report the results for a single split. Some communities exhibited more contracted curves along the y-axis, i.e. for the larger areas sampled $\beta_{\text {SOR }}$ was $<0.2$ (e.g. SCRT, MBBA, and NPGO), whereas for others it was $\sim 0.4-0.8$ (e.g. RLS_F, RLS_I, and FIA). $\beta_{\text {SIM }}$ exhibited a similar pattern, representing the largest portion of $\beta$-diversity across the scale gradient and for all the communities. $\beta_{\mathrm{NES}}$ seemed to be relatively insensitive to changes in area sampled, and always had a smaller contribution to $\beta$-diversity, although it increased slightly for some communities as area increased (Fig. 2). The results were qualitatively very similar when using area sampled as grain * number of samples (Fig. S3). Model fitting indicated that a power law always provided the best fit according to AIC (Fig. S4). Moreover, the estimated power law coefficients fell in a relatively narrow parameter space (Fig. S5), and in many situations were not statistically different between datasets.

\section{Pairwise $\beta$-diversity}

We used a single random trial since there was relatively small variability in the estimated coefficients among trials, and restricted our comparisons to the lowest levels of the scale gradient. At a given level, $\beta_{\text {sor }}$ increased with geographic distance, and as the scale level increased overall dissimilarity decreased (Figs. 3 and S6). While $\beta_{\text {sim }}$ followed $\beta_{\text {sor }}$ patterns closely, $\beta_{\text {nes }}$ exhibited significant negative slopes at the grain level, and did not show a consistent trend across the other levels, but always exhibited shallower 
slopes (Figs. 3 and S6). In general, pairwise-vs-distance slopes remained relatively unchanged as grain decreased, and for several datasets were not different between scaling levels, while intercepts were consistently higher comparing grain $v s 1 / 16$ and $v s 1 / 8$ for $\beta_{\text {sor }}$ and for $\beta_{\text {sim }}$ (Figs. 3 and 4 ; note the different scales for slopes and intercepts and across the metrics). For $\beta_{\text {nes }}$, grain had no effect for both parameters (Fig. 3). Turnover accounted for the largest portion of $\beta$-diversity across all the communities and the scale gradient (Fig. S6). Pairwise comparisons between the bootstrapped coefficients for $\beta_{\text {sim }}$ and $\beta_{\text {nes }}$ showed that both slopes and intercepts were consistently higher for turnover (Fig. 4).

\section{Discussion}

Our study - the first comprehensive empirical analysis of $\beta$-diversity scaling patterns - reveals a remarkable consistency across taxa, with multiple-site $\beta$-diversity decreasing with area sampled according to a power law. Furthermore, for pairwise comparisons, the rates of increase of dissimilarity with distance remained largely unchanged across scales spanning several orders of magnitude, while grain affects overall dissimilarity values. In both analyses, turnover accounted for most of $\beta$-diversity across all the communities, showing similar scaling properties to total dissimilarity, with the nestedness component being lower and relatively insensitive to large changes in grain. In general, the smaller the area sampled (and for more dispersal limited organisms), the more dissimilar the sites being compared will be (Nekola and White 1999, Mac Nally et al. 2004, Barton et al. 2013). This is consistent with what we found in both our analyses: a decrease in multiple-site dissimilarity as grain increased, and higher overall pairwise dissimilarity for smaller grains (consistently higher intercepts for lower levels of the scale gradient). As grain size increases, more species are shared between the sampled areas, and micro-environmental differences are attenuated. Moreover, larger areas will harbour more species and pooling samples to obtain coarser grains results in larger samples and consequently increased probability of sampling more rare species.

Multiple-site $\beta$-diversity decreases as a power law of sampled area for all the communities analysed, when some variability could be expected due to specific ecological and/or environmental underlying factors (Barton et al. 2013). In addition, both types of $\beta$-diversity exhibited regular patterns, revealing a 
remarkable consistency in $\beta$-diversity scaling properties across taxa. The datasets analysed comprise very different taxa, with different ecological and dispersal characteristics. Moreover, the datasets analysed cover a wide range of spatial extents, grain sizes and species richness, suggesting that our results are robust to large variation in these fundamental aspects of ecological studies. Our findings can be related to two fundamental patterns of ecology: species spatial aggregation patterns and the triphasic SAR, which we discuss in turn.

One of the three assumptions common across unified biodiversity theories is that conspecific individuals are spatially aggregated (McGill 2010b). Spatial aggregation has been particularly studied for terrestrial plants and at smaller spatial scales (Condit et al. 2000, 2002, Plotkin and Muller-Landau 2002, Plotkin et al. 2002, Morlon et al. 2008). Conspecific aggregation affects the expected similarity between samples within a regional landscape (Plotkin and Muller-Landau 2002) and DDR rates (Morlon et al. 2008). Our results suggest that conspecific aggregation is a plausible mechanism across spatial scales spanning several orders of magnitude, from very small local samples to very large regional areas, and across taxonomic groups. However, two further aspects could be relevant in framing our results and the effects of species spatial aggregation. First, Morlon et al. (2008) noted that the homogeneous Poisson-cluster Process was unable to completely reproduce distance-decay relationships in empirical forest plots, particularly for more heterogeneous landscapes. This process assumes a single scale of aggregation, a constant density of conspecifics across the landscape, and ignores interspecific spatial correlations; these assumptions are likely violated in natural systems. Second, we found a different functional form of the expected similarity with increasing area to that derived by Plotkin \& Muller-Landau (2002). Given the consistency of the $\beta$-diversity scaling patterns we found and the range of scales analysed (and presumably the high habitat heterogeneity underlying the scale gradient), together with the two above inconsistencies, our results suggest that neither the negative binomial nor a Poisson-cluster Process will be able to adequately describe (dis)similarity patterns across scales (from local to regional scales). We suggest that such inability may be attributable to the underlying assumption of independent species placement (i.e. species randomly distributed regardless of other species), which is unlikely to hold across scales. Morlon et al. (2008) also suggested that interspecific spatial aggregation could potentially affect distance-decay relationships by indirectly influencing species abundances and intraspecific aggregation, while noting that the inability of the Poisson Cluster model in reproducing empirical patterns would reveal the need of 
incorporating more heterogeneity into the models. We suggest that inter-species aggregation is also a relevant driver of the patterns we found, particularly at larger spatial scales.

SARs describe the increase in species richness as area sampled increases. In logarithmic space, SARs are concave for small scales (downward accelerating), become linear for intermediate scales, and then convex for larger scales (upward accelerating), indicating that distinct regions are then being sampled (Rosenzweig 1995). Intuitively, the transition between these distinct phases (i.e. inflection points) can be related to the dispersal ability of the taxa studied. Since in all our datasets the total extent sampled was much larger than the areas at which the inflection point indicates progressively increasing slopes (Fig. S7), one can expect relatively high $\beta$-diversity (mainly due to turnover) even for very large samples (e.g. the bisection level), since each section is likely to contain some species not present in the other section. In other words, if the total spatial extent roughly coincided with the inflection point, the two random bisections would more likely contain the entire set of species present in the region, and $\beta$-diversity would be $\sim 0$. Our results are consistent with this expectation. Furthermore, the dataset with the largest difference between the inflection point and the overall extent is RLS Invertebrates (RLS_I), which exhibited the overall highest $\beta$-diversity values across the scale gradient. Moreover, of the datasets analysed, RLS Invertebrates is arguably the most dispersal-constrained community, further suggesting that both dispersal ability and spatial extent underlie the patterns found. Nonetheless, we have not explicitly explored how dispersal ability could be linked to our results, thus further research is still necessary to evaluate which species traits underlie the patterns found, and furthermore if such patterns would be consistent for other taxa and ecosystems. Overall, our results suggest the spatial extent (and potentially the underlying habitat heterogeneity), dispersal abilities, and both intra- and inter-species aggregation patterns as important drivers of $\beta$-diversity scaling patterns.

\section{Multiple-site $\beta$-diversity}

There were some differences among the scaling curves, namely a contraction along the $y$-axis for some communities. In other words, some communities still showed very high multiple-site $\beta$-diversity even for very large sampled areas. $\beta_{\mathrm{SOR}}$ at the bisection level was particularly high for the RLS Fish and Invertebrates (RLS_F; RLS_I), and FIA datasets (Fig. 2). For more heterogeneous landscapes and for 
organisms with lower dispersal ability, sampled areas are expected to be more dissimilar (Qian 2009, Si et al. 2015). While the spatial configuration of the RLS data could also potentially affect multiple-site $\beta$ diversity patterns, and we have not tested for this effect (sampling sites distributed around Australia vs contiguous cloud of more or less dispersed sampling locations across the spatial extent), the FIA dataset exhibited similar patterns, which lends support to the argument that ecological properties of the different taxa, rather than the spatial distribution of the individual sampling locations, more strongly affected multiple-site $\beta$-diversity patterns in our analysis, along with the influence of the overall extent sampled, as discussed above.

\section{Pairwise $\beta$-diversity}

The rates of pairwise-vs-distance relationships remained relatively unchanged as grain decreased, across very large ranges of area sampled, while intercepts increased with decreasing grain size. These results contrast with previous reports (Keil et al. 2012, Steinbauer et al. 2012), but are in accordance with Morlon et al.'s (2008) results showing that grain affected overall similarity values, rather than DDR rates, except at the smallest sampled area. Our results are consistent with Morlon et al.'s, although we found no significant differences among the scaling levels for some of the datasets (Fig. 4). However, the range of areas investigated differs greatly between our studies: grain values ranged between $0.0004-6.25$ ha in Morlon et al., while our scale gradient spanned much larger areas, with a very sharp increase from the smallest to the subsequent levels. Since Morlon et al.'s range is much narrower, we can reconcile both studies in that DDR rates seem to be overall robust to large variations in area sampled, while those variations more strongly affect overall dissimilarity (which is also aligned with Keil et al.'s results). This suggests that DDR rates alone are not a good indicator of compositional patterns across scales, while intercepts provide a better assessment of dissimilarity across spatial scales (Morlon et al. 2008, GómezRodríguez and Baselga 2018), as do multiple-site dissimilarity metrics.

Our results differ from those of Keil et al. (2012) and Steinbauer et al. (2012), but we attribute this to the very different spatial frameworks between our studies. Firstly, both grain and extent were allowed to vary in those studies. Moreover, in Steinbauer et al.'s analysis, the distance between plots was kept constant while increasing plot size, and slopes were more strongly affected by varying extent than grain size. In 
our analysis, we used a fixed extent, so that community data was sampled from a fixed species pool, while the areas sampled and the distance between them were unconstrained. This isolates variation in $\beta$ diversity from variation in gamma diversity. Secondly, we did not impose static grids over regional or continental extents, but analysed dissimilarity patterns across a scale gradient spanning several orders of magnitude. Finally, we used data collected with a high degree of spatial resolution, from many small representative samples, rather than incidence data across large grid sizes, atlases or simulated data. However, one caveat of our study was that we were unable to fully explore the scale gradient for the pairwise-vs-distance analysis, as we could not include the higher levels of the scale gradient. Nonetheless, our results showed that large increases in grain had no or little effect on DDR rates, but did affect overall dissimilarity; i.e. grain size, rather than the distance between sites, more strongly affected dissimilarity patterns across the scale gradient.

Higher intercepts indicate higher $\beta$-diversity, since even closer locations exhibit low similarity; this can be related to higher habitat heterogeneity in those communities, and/or linked to low dispersal abilities of the organisms (Nekola and White 1999, Morlon et al. 2008). Both multiple-site and DDR intercepts are good indicators of how dissimilarity is affected by sampling grain. For some datasets, DDR slopes differed more between scaling levels, namely for the RLS datasets and SCRT. The RLS survey datasets are the two most diverse in our analysis in terms of species richness; moreover, the sampling locations are distributed around Australia, which encompasses distinct ecoregions. Thus, one could expect the rates of dissimilarity between very small sample sizes at the grain level to differ to grain sizes orders of magnitude larger. On the other extreme, SCRT is the dataset with fewer species, so sampling effects (e.g. not sampling the rarest species) could explain the differences in DDR rates. Nonetheless, the overall pattern across all the datasets is that grain affected intercepts more strongly than slopes, suggesting that including information about variation in intercepts (i.e. dissimilarity at the smallest scale) is fundamental to understanding dissimilarity patterns (Morlon et al. 2008, Gómez-Rodríguez and Baselga 2018).

\section{$\beta$-diversity components}

Partitioning $\beta$-diversity into turnover and nestedness revealed that the two components exhibit divergent spatial patterns, with turnover strongly driving total $\beta$-diversity patterns, for all the communities and across the scale gradient (see also Soininen et al. 2017). In contrast, the nestedness component 
contribution was systematically low and generally scale insensitive. Since turnover followed the total dissimilarity pattern very closely, the nestedness component had to exhibit a contrasting pattern, due to the additive nature of the partitioning framework (Baselga 2010). Interestingly, nestedness decreased with geographic distance only at the lowest level of the scale gradient (and occasionally also at the next level), being seemingly unaffected when calculating dissimilarity between sections at higher levels. It is not straightforward to establish expectations for how the nestedness component should behave across scales, since several interacting factors must be considered. For instance, the relative balance between turnover and nestedness depends both on dispersal rates and on environmental heterogeneity (Gianuca et al. 2017). Complementary analyses including dispersal ability and habitat heterogeneity might provide further insights to help discern how the two components are expected to behave (Soininen et al. 2017). We note here that although $\beta$-diversity partitioning has sparked some debate (Almeida-Neto et al. 2012, Carvalho et al. 2012), the framework used here was shown to adequately measure the portions of dissimilarity derived exclusively from species replacement and nested patterns (Baselga 2012, Baselga and Leprieur 2015).

The fact that species replacement, rather than change in species richness, was the main driver of compositional change across the scale gradient offers important insights for the underlying processes driving $\beta$-diversity at different spatial scales. It also has implications for both our understanding of spatiotemporal dynamics of biodiversity change and conservation (Baselga 2010, Dornelas et al. 2014, McGill et al. 2015, Magurran et al. 2015, Socolar et al. 2016), highlighting that substantial changes in community composition might be occurring, despite species richness remaining seemingly stable. A recent meta-analysis by Soininen et al. (2017) also found turnover to be the dominant component of $\beta$ diversity across different latitudes, spatial extents and taxa. A general understanding of the relative contribution of each component to $\beta$-diversity patterns across spatial scales has relevant implications for conservation strategies, specifically concerning the number and size of areas to be protected depending on whether species turnover or species richness differences are dominant (Baselga 2010, Socolar et al. 2016). Our study represents the first investigation of turnover and nestedness components contributions independent of latitudinal, longitudinal or environmental gradients, revealing consistent patterns for the communities analysed.

'This article is protected by copyright. All rights reserved.' 
Conclusions

We found highly regular $\beta$-diversity scaling patterns across the communities analysed, with multiple-site dissimilarity decreasing with grain according to a power law, and with turnover being the main driver of $\beta$-diversity across scales spanning several orders of magnitude. We suggest that the scaling patterns found are likely underpinned by the interplay of species dispersal abilities, intra- and inter-specific spatial aggregation patterns, as well as the overall diversity being sampled (as exemplified by the potential link with the SAR). Given the current need to quantify how biodiversity is changing in the Anthropocene, it is of critical importance that we understand how spatial scale can influence changes regarding community structure and composition. Our findings provide valuable insights to understanding and synthetizing $\beta$ diversity scaling patterns across taxa and ecosystems.

\section{Acknowledgements}

We are grateful to two anonymous reviewers and Andrés Baselga for insightful comments. We are grateful to all the scientists, data collectors and their funders for making data publicly available. We thank the University of St Andrews Bioinformatics Unit (Wellcome Trust ISSF grant 105621/Z/14/Z). L.H.A. was supported by Fundação para a Ciência e Tecnologia, Portugal (POPH/FSE SFRH/BD/90469/2012), A.E.M. by the ERC BioTIME (250189) and BioCHANGE (727440), and B.J.M. by USDA Hatch grant to MAFES \#1011538 and NSF ABI grant \#1660000. The BioTIME database was funded by ERC AdG BioTIME (250189) and ERC PoC BioCHANGE (727440). Faye Moyes assisted with data management. All the inset icons in the Figures are from the Noun Project under CC license: Fish by Jens Tärning; Bird by Ana María Lora Macias; Shell by AFY Studio; Anemonia anemone by Vega Asensio, Starfish by Star and Anchor Design; and Tree by Mister Pixel.

\section{Author contributions}

L.H.A. and M.D. designed the study, L.H.A. assembled the data and performed all the analyses. L.H.A. wrote the first draft of the manuscript; all authors have discussed the results and contributed extensively to the final manuscript. 
Data Accessibility statement: All the datasets used in this study can be accessed through either the BioTIME database or the EcoData Retriever, or alternatively from the original sources cited. 


\section{References}

Almeida-Neto, M. et al. 2012. Rethinking the relationship between nestedness and beta diversity: a comment on Baselga (2010). - Glob. Ecol. Biogeogr. 21: 772-777.

Anderson, M. J. et al. 2011. Navigating the multiple meanings of $\beta$ diversity: a roadmap for the practicing ecologist. - Ecol. Lett. 14: 19-28.

Barton, P. S. et al. 2013. The spatial scaling of beta diversity. - Glob. Ecol. Biogeogr. 22: 639-647.

Baselga, A. 2010. Partitioning the turnover and nestedness components of beta diversity. - Glob. Ecol. Biogeogr. 19: 134-143.

Baselga, A. 2012. The relationship between species replacement, dissimilarity derived from nestedness, and nestedness. - Glob. Ecol. Biogeogr. 21: 1223-1232.

Baselga, A. and Orme, C. D. L. 2012. Betapart: An R package for the study of beta diversity. - Methods Ecol. Evol. 3: 808-812.

Baselga, A. and Leprieur, F. 2015. Comparing methods to separate components of beta diversity. Methods Ecol. Evol. 6: 1069-1079.

Baselga, A. et al. 2017. betapart: Partitioning Beta Diversity into Turnover and Nestedness Components. R package version 1.4-1.

Bivand, R. and Rundel, C. 2016. rgeos: Interface to Geometry Engine - Open Source (GEOS). R package version 0.3-19.

Brown, S. K. R. et al. 2005. "East Coast North America Strategic Assessment Groundfish Atlas ECNASAP.” - OBIS Canada, Bedford Inst. Oceanogr. Dartmouth, Nov. Scotia, Canada. Available at: http://iobis.org/, accessed 2013.

Carvalho, J. C. et al. 2012. Determining the relative roles of species replacement and species richness differences in generating beta-diversity patterns. - Glob. Ecol. Biogeogr. 21: 760-771.

Condit, R. S. et al. 2000. Spatial patterns in the distribution of tropical tree species. - Science 288: 14141418.

Condit, R. et al. 2002. Beta-diversity in tropical forest trees. - Science 295: 666-669.

DATRAS 2010a. "Fish trawl survey: ICES North Sea International Bottom Trawl Survey for commercial fish species. ICES Database of trawl surveys (DATRAS).” - Int. Counc. Explor. Sea, Copenhagen. Available at: http://www.emodnet-biology.eu/data-catalog??module=dataset\&dasid=2763, accessed 2013.

'This article is protected by copyright. All rights reserved.' 
DATRAS 2010b. "Fish trawl survey: Irish Ground Fish Survey for commercial fish species. ICES Database of trawl surveys (DATRAS).” - Int. Counc. Explor. Sea, Copenhagen. Available at: http://www.emodnet-biology.eu/data-catalog?module=dataset\&dasid=2762, accessed 2013.

Dornelas, M. et al. 2014. Assemblage Time Series Reveal Biodiversity Change but Not Systematic Loss. - Science 344: 296-299.

Dornelas, M. et al. 2018. BioTIME: A database of biodiversity time series for the anthropocene. - Glob. Ecol. Biogeogr. 27: 760-786.

Edgar, G. J. and Stuart-Smith, R. D. 2008. "Reef Life Survey (RLS): Invertebrates.” - Inst. Mar. Antarct. Stud. Available at: http://catalogue-

rls.imas.utas.edu.au/geonetwork/srv/en\%0A/metadata.show?uuid=60978150-1641-11dd-a32600188b4c0af, accessed 2016.

Edgar, G. J. and Stuart-Smith, R. D. 2014a. "Reef Life Survey (RLS): Global reef fish dataset.” - Inst. Mar. Antarct. Stud. Available at: http://catalogue-

rls.imas.utas.edu.au/geonetwork/srv/en\%0A/metadata.show?uuid=9c766140-9e72-4bfb-8f04d51038355c5, accessed 2016.

Edgar, G. J. and Stuart-Smith, R. D. 2014b. Systematic global assessment of reef fish communities by the Reef Life Survey program. - Sci. Data 1: 140007.

Gaston, K. J. et al. 2007. The scaling of spatial turnover: pruning the thicket. - In: Storch, D. et al. (eds), Scaling Biodiversity. Cambridge University Press, pp. 181-222.

Gianuca, A. T. et al. 2017. Effects of dispersal and environmental heterogeneity on the replacement and nestedness components of $\beta$-diversity. - Ecology 98: 525-533.

Gómez-Rodríguez, C. and Baselga, A. 2018. Variation among European beetle taxa in patterns of distance decay of similarity suggests a major role of dispersal processes. - Ecography. in press. doi:10.1111/ecog.03693

Harrison, S. et al. 1992. Beta Diversity on Geographic Gradients in Britain. - J. Anim. Ecol. 61: 151-158.

Harte, J. et al. 2009. Biodiversity scales from plots to biomes with a universal species-area curve. - Ecol. Lett. 12: 789-797.

Keil, P. et al. 2012. Patterns of beta diversity in Europe: the role of climate, land cover and distance across scales. - J. Biogeogr. 39: 1473-1486.

Koleff, P. et al. 2003. Measuring beta diversity for presence -absence data. - J. Anim. Ecol. 72: 367-382. 
Leibold, M. A. et al. 2004. The metacommunity concept: a framework for multi-scale community ecology. - Ecol. Lett. 7: 601-613.

Levin, S. 1992. The problem of pattern and scale in ecology: the Robert H. MacArthur award lecture. Ecology 73: 1943-1967.

Mac Nally, R. et al. 2004. Comparative influence of spatial scale on beta diversity within regional assemblages of birds and butterflies. - J. Biogeogr. 31: 917-929.

MacArthur, R. H. 1972. Geographical ecology. - Princeton University Press.

Magurran, A. E. et al. 2015. Rapid biotic homogenization of marine fish assemblages. - Nat. Commun. 6 : 8405.

McGill, B. J. 2010a. Matters of scale. - Science 328: 575-576.

McGill, B. J. 2010b. Towards a unification of unified theories of biodiversity. - Ecol. Lett. 13: 627-642.

McGill, B. J. et al. 2015. Fifteen forms of biodiversity trend in the anthropocene. - Trends Ecol. Evol. 30: $104-113$.

McGlinn, D. and White, E. 2015. ecoretriever: R Interface to the EcoData Retriever. R package version 0.2 .1 .

Morlon, H. et al. 2008. A general framework for the distance-decay of similarity in ecological communities. - Ecol. Lett. 11: 904-917.

Morris, B. D. and White, E. P. 2013. The EcoData Retriever: Improving Access to Existing Ecological Data. - PLoS One 8: e65848.

NatureCounts (a) "Maritimes Breeding Bird Atlas (2006-2010): point count data." - NatureCounts, a node Avian Knowl. Network. Bird Stud. Canada. Available at: http://www.birdscanada.org/birdmon/, accessed 2012.

NatureCounts (b) “Ontario Breeding Bird Atlas (2001-2005): point count data.” - NatureCounts, a node Avian Knowl. Network. Bird Stud. Canada. Available at: http://www.birdscanada.org/birdmon/, accessed 2012.

Nekola, J. C. and White, P. S. 1999. The distance decay of similarity in biogeography and ecology. - J. Biogeogr. 26: 867-878.

Nekola, J. C. and McGill, B. J. 2014. Scale dependency in the functional form of the distance decay relationship. - Ecography 37: 309-320.

North Pacific Groundfish Observer Program "North Pacific Groundfish Observer.” - Alaska Fish. Sci. 
Cent. Available at: http://iobis.org/, accessed 2013.

Pardieck, K. L. et al. 2016. "North American Breeding Bird Survey Dataset 1966 - 2015, version 2015.1." - U.S. Geol. Surv. Patuxent Wildl. Res. Cent. Available at: www.pwrc.usgs.gov/BBS/RawData/, accessed 2016.

Pebesma, E. J. and Bivand, R. S. 2005. Classes and methods for spatial data in R. R News 5 (2), http://cran.r-project.org/doc/Rnews/.

Pinheiro, J. et al. 2016. nlme: Linear and Nonlinear Mixed Effects Models. R package version 3.1-128.

Plotkin, J. B. and Muller-Landau, H. C. 2002. Sampling the Species Composition of a Landscape. Ecology 83: 3344-3356.

Plotkin, J. B. et al. 2002. Cluster analysis of spatial patterns in Malaysian tree species. - Am. Nat. 160: 629-644.

Preston, F. 1948. The commonness, and rarity, of species. - Ecology 29: 254-283.

Qian, H. 2009. Global comparisons of beta diversity among mammals, birds, reptiles, and amphibians across spatial scales and taxonomic ranks. - J. Syst. Evol. 47: 509-514.

R Core Team 2017. R: A language and environment for statistical computing. R Foundation for Statistical Computing, Vienna, Austria. http://www.R-project.org/.

Ricklefs, R. 1987. Community diversity: relative roles of local and regional processes. - Science 235: $167-171$.

Rosenzweig, M. L. 1995. Species Diversity in Space and Time. - Cambridge University Press.

Schuler, M. S. et al. 2017. Habitat patch size alters the importance of dispersal for species diversity in an experimental freshwater community. - Ecol. Evol. 7: 5774-5783.

Si, X. et al. 2015. Revealing Beta-Diversity Patterns of Breeding Bird and Lizard Communities on Inundated Land-Bridge Islands by Separating the Turnover and Nestedness Components. - PLoS One 10: e0127692.

Socolar, J. B. et al. 2016. How Should Beta-Diversity Inform Biodiversity Conservation? - Trends Ecol. Evol. 31: 67-80.

Soininen, J. et al. 2017. A meta-analysis of nestedness and turnover components of beta diversity across organisms and ecosystems. - Glob. Ecol. Biogeogr. 27: 96-109.

Steinbauer, M. J. et al. 2012. Current measures for distance decay in similarity of species composition are influenced by study extent and grain size. - Glob. Ecol. Biogeogr. 21: 1203-1212. 
Storch, D. et al. 2012. Universal species-area and endemics-area relationships at continental scales. Nature 488: 78-81.

Svenning, J.-C. et al. 2011. Climate, history and neutrality as drivers of mammal beta diversity in Europe: insights from multiscale deconstruction. - J. Anim. Ecol. 80: 393-402.

Tonkin, J. D. et al. 2016. Contrasting metacommunity structure and beta diversity in an aquatic-floodplain system. - Oikos 125: 686-697.

Tuomisto, H. 2010a. A diversity of beta diversities: straightening up a concept gone awry. Part 2. Quantifying beta diversity and related phenomena. - Ecography 33: 23-45.

Tuomisto, H. 2010b. A diversity of beta diversities: straightening up a concept gone awry. Part 1. Defining beta diversity as a function of alpha and gamma diversity. - Ecography 33: 2-22.

Ulrich, W. et al. 2016. The tangled link between $\beta$ - and $\gamma$-diversity: a Narcissus effect weakens statistical inferences in null model analyses of diversity patterns. - Glob. Ecol. Biogeogr. 26: 1-5.

USDA Forest Service 2010. Forest inventory and analysis national core field guide (Phase 2 and 3). Version 4.0. USDA Forest Service, Forest Inventory and Analysis, Washington, D.C., USA.

USFS “Landbird Monitoring Program (UMT-LBMP).” - US For. Serv. Available at: http://www.avianknowledge.net/, accessed 2012.

Wade, E. J. 2011. "Snow crab research trawl survey database (Southern Gulf of St. Lawrence, Gulf region, Canada) from 1988 to 2010.” - OBIS Canada Digit. Collect. OBIS Canada, Bedford Inst. Oceanogr. Dartmouth, Nov. Scotia, Canada. Available at: http://iobis.org/, accessed 2013.

Wen, Z. et al. 2016. Multiscale partitioning of small mammal $\beta$-diversity provides novel insights into the Quaternary faunal history of Qinghai-Tibetan Plateau and Hengduan Mountains. - J. Biogeogr. 43: $1412-1424$.

Whittaker, R. H. 1960. Vegetation of the Siskiyou Mountains, Oregon and California. - Ecol. Monogr. 30: $279-338$.

Wiens, J. A. 1989. Spatial Scaling in Ecology. - Funct. Ecol. 3: 385-397.

Williams, C. B. 1943. Area and number of species. - Nature 152: 264-267.

Woudenberg, S. W. et al. 2010. The Forest Inventory and Analysis Database: Database description and users manual version 4.0 for Phase 2. Gen. Tech. Rep. RMRSGTR-245.

Zacaï, A. et al. 2016. Gauging scale effects and biogeographical signals in similarity distance decay analyses: an Early Jurassic ammonite case study. - Palaeontology 59: 671-687. 


\section{Figure Legends}

Figure 1. Schematic representation of the scale gradient, showing an example of how an encompassing circle was drawn and a random point was selected to establish bisections ( $a$ and $b$ ) and thirds $(c-e)$. For multiple-site $\beta$-diversity scaling curves, the smallest section area at each level was used (b and c); for pairwise $\beta$-diversity, the distances between the centroids of each section were calculated ( $\mathrm{d}$ and $\mathrm{e})$.
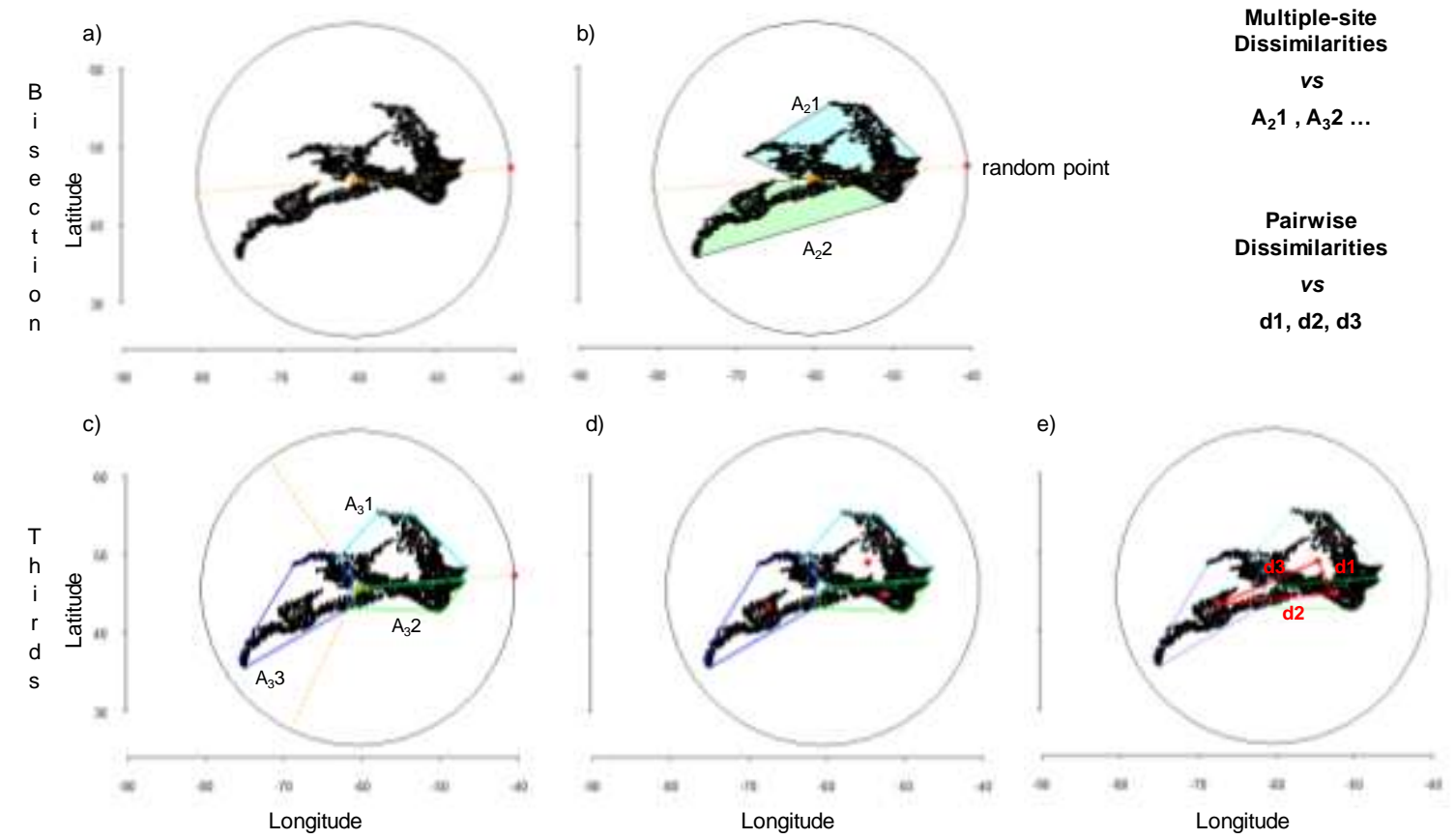

'This article is protected by copyright. All rights reserved.' 
Figure 2. Multiple-site $\beta$-diversity scaling curves, showing the decrease of $\beta_{\text {SOR }}$ and the turnover component $\beta_{\text {SIM }}$ with increasing grain according to a power law (on a semi-log plot). The points represent the multiple-site dissimilarity values for each level of the scaling gradient $(1 / 2,1 / 3,1 / 4,1 / 8,1 / 16$ of the total extent; and sampling grain), where the bisection level has the largest area, and the grain size represents the smallest area. The lines represent the power law model fitted to each dataset for each dissimilarity metric, which provided a better fit than a linear logit model according to AIC (Fig. S4). The area plotted is the minimum convex hull polygon value at each level; Fig. S3 shows the scaling curves with area calculated as grain * number of samples.
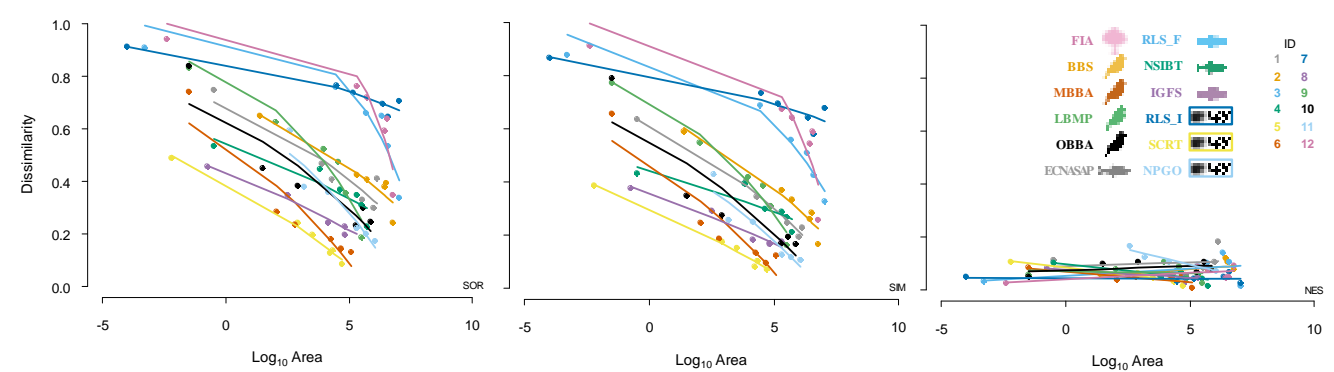
Figure 3. Pairwise dissimilarities $v s$ distance negative exponential models parameters ((a) slopes and (b) intercepts) as a function of the scaling level, combining all the random splitting trials and datasets; note the differences in the y-axis scales for the two parameters. For this analysis, we explored only three scaling levels (grain; 1/16; and 1/8) because the higher levels in the scale gradient contain too few pairwise comparisons to confidently estimate DDR.

a)

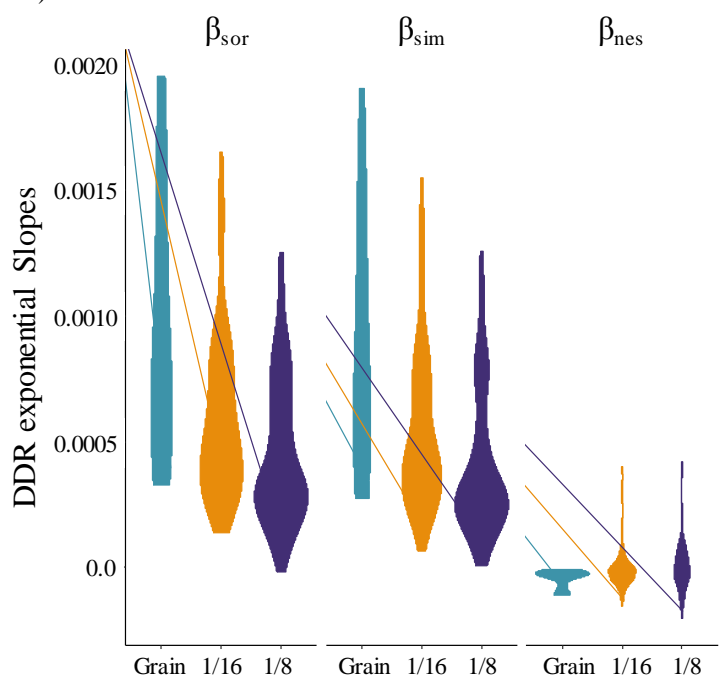

b)

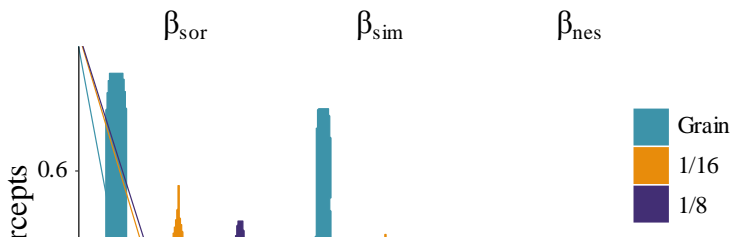

Scaling level 
Figure 4. Bootstrapped distributions of the negative exponential models parameters used to describe pairwise dissimilarities as a function of distance for $\beta_{\text {sim }}$ and $\beta_{\text {nes }}$ (note the differences in the $\mathrm{x}$-axis scales between slopes and intercepts and between metrics). Each row is identified by the corresponding dataset abbreviation as in Table 1

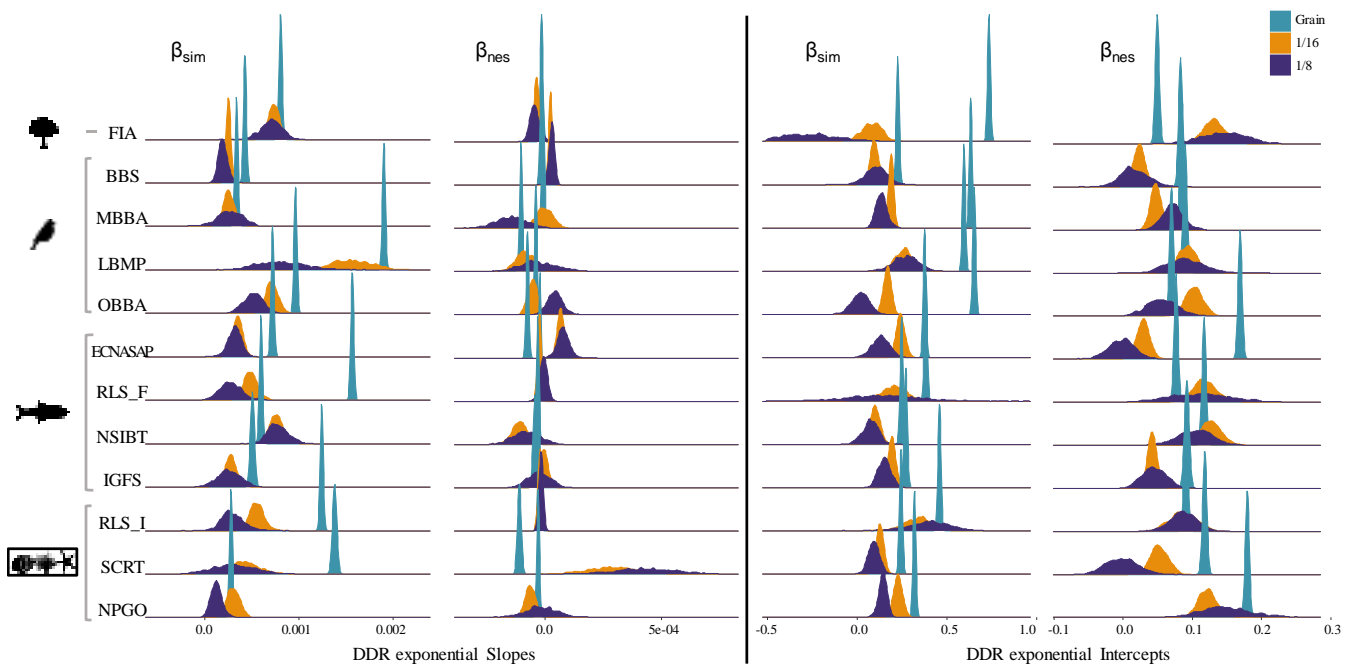




\section{Table Legend}

Table 1 - Community data with the corresponding taxon, species richness, spatial extent, grain and data source.

\begin{tabular}{|c|c|c|c|c|c|c|c|c|}
\hline Dataset Title & Abbreviation & Taxon & Usage notes & $\begin{array}{l}\text { Spatial extent } \\
\left(\mathrm{km}^{2}\right)\end{array}$ & Grain $\left(\mathrm{km}^{2}\right)$ & $\begin{array}{c}\text { Number of } \\
\text { species }\end{array}$ & $\begin{array}{c}\text { Number of } \\
\text { samples }\end{array}$ & Reference \\
\hline Forest Inventory Analysis & FIA & Trees & $\begin{array}{l}\text { 2013; excluded } \\
\text { Alaska }\end{array}$ & 16663141 & 0.004047 & 305 & $19427^{¥}$ & $\begin{array}{c}\text { (USDA Forest Service } \\
\text { 2010, Woudenberg et } \\
\text { al. 2010) }\end{array}$ \\
\hline $\begin{array}{c}\text { North American Breeding Bird } \\
\text { Survey }\end{array}$ & BBS & Birds & $\begin{array}{l}\text { 2015; USA data only } \\
\text { (excluded Alaska) }\end{array}$ & 13104786 & 25.42715 & 521 & 2420 & (Pardieck et al. 2016) \\
\hline Maritimes Breeding Bird Atlas & MBBA & Birds & 2009 & 480235 & $0.031416^{*}$ & 163 & 3243 & (NatureCounts a) \\
\hline Landbird Monitoring Program & LBMP & Birds & 2004 & 1057570 & $0.031416^{*}$ & 229 & $5107^{¥}$ & (USFS) \\
\hline Ontario Breeding Bird Atlas & OBBA & Birds & 2003 & 3545420 & 0.031416 & 233 & $19611^{¥}$ & (NatureCounts b) \\
\hline $\begin{array}{c}\text { East Coast North America Strategic } \\
\text { Assessment }\end{array}$ & ECNASAP & Fish & 1994 & 7229693 & 0.33336 & 110 & 2101 & (Brown et al. 2005) \\
\hline $\begin{array}{c}\text { Reef Life Survey: Global reef fish } \\
\text { dataset }\end{array}$ & RLS_F & Fish & $\begin{array}{c}\text { Spatial subset } \\
\text { around Australia }\end{array}$ & 572747 & 0.0005 & 1847 & $6666^{¥}$ & $\begin{array}{l}\text { (Edgar and Stuart- } \\
\text { Smith 2014a, b) }\end{array}$ \\
\hline $\begin{array}{l}\text { ICES North Sea International } \\
\text { Bottom Trawl Survey for } \\
\text { commercial fish species }\end{array}$ & NSIBT & Fish & 2011 & 2726171 & $0.33336^{*}$ & 131 & 688 & (DATRAS 2010a) \\
\hline $\begin{array}{l}\text { Irish Ground Fish Survey for } \\
\text { commercial fish species }\end{array}$ & IGFS & Fish & 2004 & 967879 & 0.177792 & 207 & 163 & (DATRAS 2010b) \\
\hline Reef Life Survey: Invertebrates & RLS_I & Invertebrates & $\begin{array}{c}\text { Spatial subset } \\
\text { around Australia }\end{array}$ & 572747 & 0.0001 & 1013 & $6817^{¥}$ & $\begin{array}{l}\text { (Edgar and Stuart- } \\
\text { Smith 2008, 2014b) }\end{array}$ \\
\hline $\begin{array}{c}\text { Snow crab research trawl survey } \\
\text { database }\end{array}$ & SCRT & Benthos & 2009 & 167455 & 0.00642 & 32 & 354 & (Wade 2011) \\
\hline North Pacific Groundfish Observer & NPGO & Benthos & 1993 & 6794596 & 400 & 220 & 1007 & $\begin{array}{c}\text { (North Pacific } \\
\text { Groundfish Observer }\end{array}$ \\
\hline
\end{tabular}

*Grain was approximated to similar studies; ${ }^{*}$ indicates 2000 random samples were used for the grain level pairwise dissimilarities analysis.

Program)

'This article is protected by copyright. All rights reserved.' 